\title{
Isolamento colturale e molecolare di Chlamydophila pneumoniae da pazienti con artropatie. Prospettive patogenetiche e diagnostiche
}

\author{
Margherita Giuliodori, Silva Seraceni, Veronica Biondin, Rosario Cultrera, Carlo Contini
}

Sezione di Malattie Infettive, Dipartimento di Medicina Clinica e Sperimentale, Università di Ferrara

Key words: Chlamydia pneumoniae, arthritis, synovial fluid, Colture, PBMC, DNA, PCR, RT-PCR

Coltural and molecular isolation of Chlamydophila pneumoniae from patients with joint disease. Pathogenic and diagnostic perspectives.

\section{SUMMARY}

Chlamydophila pneumoniae is an ubiquitous intracellular pathogen which causes acute respiratory diseases and may be associated with chronic inflammatory diseases including atherosclerosis, multiple sclerosis and arthritis. C. pneumoniae is rarely cultured from the synovial fluid or blood, and serology is seldom useful. So far most of the studies concerning the possible association between $C$. pneumoniae and arthritis have been made by molecular methods. Recent advances in the standardization of polymerase chain reaction techniques have shown to confirm a role of $C$. pneumoniae not only in reactive arthritis (ReA) but also in chronic arthritis. In this study, we investigated whether $C$. pneumoniae could be isolated in synovial fluid and PBMC specimens of patients with different forms of arthritis including ReA. Advanced PCR and Reverse transcriptase PCR techniques targeting different chlamydial genes associated to a novel culture method based on combination of additional centrifugation and extension of culture time, were applied to detect $C$. pneumoniae in 6 patients with chronic synovitis including one with Anchylosing Spondylitis and relapsing joint swelling. For this patient, serological, coltural as well as molecular assays did detect C. pneumoniae only. Particularly, a high expression of Heat shock protein 60 and 70 of $C$. pneumoniae was found in the PBMC and the synovial compartments, thus confirming the ability of $C$. pneumoniae to survive inside blood ad synovia in vital and metabolically active forms. By contrast, the selective decrease of MOMP and I6sRNA, leads to hypotesize a different expression of Chlamydophyla genes during the different phases of infection.

\section{INTRODUZIONE}

Negli ultimi anni la relazione tra sinoviti croniche e agenti infettivi "difficili", tra cui Chlamydophila pneumoniae, ha suscitato l'interesse di numerosi studiosi (3). L'ipotesi patogenetica del "peptide artritogenico", che prevede l'induzione di cellule T citotossiche in seguito ad una cross-reazione tra autoantigeni e antigeni batterici (17), appare particolarmente appropriata nel caso delle Artriti Reattive ( $\operatorname{ReA})$, ove tra i criteri maggiori di diagnosi rientra l'anamnesi positiva per una infezione enterica o uretrale precedente l'esordio delle manifestazioni articolari (2).

Tra i numerosi microorganismi chiamati in causa nelle ReA, Chlamydia appare essere il più comune (14); inoltre, nei monociti/macrofagi sinoviali di pazienti affetti da ReA Chlamydia-associate, sono state riscontrate forme di $C$. trachomatis in stato di persistenza (18).

Peraltro, recenti indagini molecolari sembrano confermare un ruolo di $C$. pneumoniae non solo in alcuni casi di $\operatorname{ReA}(9,11)$, ma anche in altre forme di artropatie croniche (1); tuttavia il significato di tale riscontro presenta ancora numerosi aspetti controversi.

Non esistono pareri uniformi sui test laboratoristici da utilizzare per l'identificazione di Chlamydia anche se la PCR viene ritenuta una delle metodiche più efficaci per l'individuazione del microrganismo nel liquido sinoviale (16).

Recentemente, lo sviluppo di un nuovo modello colturale (Hep2) ci ha consentito di dimostrare l'abilità di C. pneumoniae di propagarsi efficacemente all'interno di cellule mononucleate in forma vitale ed infettante (5).

Gli obiettivi di questo lavoro sono stati:

a) valutare la presenza e la vitalità di C. pneumoniae nel sangue e nel fluido sinoviale di pazienti affetti da sinovite cronica;

b) studiare l'implicazione di tale microorganismo nella patogenesi di artropatie croniche non ReA.

\section{PAZIENTI E METODI}

Il presente studio è stato condotto in collaborazione con la Sezione di Reumatologia dell'Università di Ferrara. Campioni di fluido sinoviale (SF), siero e cellule mononucleate di sangue periferico (PBMC) ottenuti da 6 pazienti 
affetti da varie forme di artropatie croniche sono stati valutati mediante metodiche colturali e biomolecolari. Di questi, un paziente, affetto da Spondilite Anchilosante (SA) HLA-B27 positiva e controllata dalla terapia (FANS e Sulfasalazina), riferiva da circa 1 anno episodi di gonidrarto recidivante: da questo, sono stati raccolti campioni sequenziali di SF, siero e PBMC; i campioni di SF, sono stati sottoposti ad indagini chimico-fisiche e microbiologiche per batteri aerobi (micobatteri inclusi), anaerobi e miceti.

Dal paziente con SA inoltre, campioni sequenziali di siero sono stati valutati mediante ELISA semi-quantitativo specie-specifico per la ricerca di immunoglobuline IgA, IgG anti-C. pneumoniae (CP-Quant, Eurospital, Trieste, Italy). Sono stati considerati positivi i sieri che mostravano valori $\mathrm{BU}$ (Binding Units) $>10$. Test sierologici sono stati anche effettuati per virus (HSV 1 e 2, CMV, Enterovirus, Rosolia), batteri (Micoplasmi, Borrelia, Bartonella, Rickettsia, Leptospira e Salmonella) e parassiti (Toxoplasma gondii).

\section{Colture}

I campioni di SF e PBMC sono stati coltivati per C.pneumoniae su cellule Hep2. Quando l'incubazione è stata prolungata per $144 \mathrm{~h}, \mathrm{a} 72,96 \mathrm{e} 120 \mathrm{~h}$, sono state eseguite centrifugazioni addizionali, con cambio del medium di coltura alla $72^{\wedge}$ ora. Le colture sono state quindi valutate in IF diretta. I sovranatanti colturali sono stati raccolti prima della fissazione delle cellule e conservati per le indagini biomolecolari. Aliquote di SF e PBMC sono state valutate in via preliminare in immunofluorescenza (IF) diretta utilizzando anticorpi monoclonali FITC-coniugati specifici per $C$. pneumoniae fornitici dall'Institut Pasteur di Parigi.

Porzioni di SF $(100 \mu 1)$ sono stati posti in terreni di coltura BDG e BDA, che metabolizzano rispettivamente il glucosio e l'arginina, opportunamente modificati per SF e mantenuti a $37^{\circ} \mathrm{C}$ per una settimana, per l'isolamento e la coltura di micoplasmi (M. pneumoniae, M. hominis, M. fermentans, M. penetrans).

\section{Analisi Molecolari}

Adottando opportune precauzioni al fine di evitare il rischio di contaminazione (12) il DNA (ed RNA per i campioni del paziente con SA) è stato estratto da SF, PBMC e sovranatanti colturali, secondo protocolli precedentemente descritti (15). I geni MOMP (Major outer membrane protein), 16s rRNA, Hsp60 (Heat shock protein) ed Hsp70 di C. pneumoniae sono stati amplificati mediante $n$-PCR ed RT-PCR utilizzando set di primers specifici $(6,8,13,4)$. Il primo ciclo della
PCR è stato effettuato mediante "Touchdown PCR" secondo Mahony (13) con modifiche da noi apportate al programma di amplificazione al fine di evitare la generazione di prodotti aspecifici (14). Per la seconda amplificazione sono stati utilizzati $5 \mu \mathrm{l}$ dei prodotti di PCR, diluiti 10 volte. Tutti i campioni sono stati testati preliminarmente per la presenza di inibitori di PCR (4). I prodotti di amplificazione sono stati quindi sottoposti ad elettroforesi su gel d'agarosio insieme a controlli positivi e controlli negativi e visualizzati mediante colorazione con bromuro d'etidio.

\section{RISULTATI}

Da 6 pazienti affetti da varie forme di artropatia cronica sono stati raccolti complessivamente $8 \mathrm{SF}$ e 12 PBMC, di cui 3 SF e 7 PBMC provenienti dal paziente con SA. Le caratteristiche dei pazienti e i risultati dello studio in PCR effettuato sui campioni freschi e sui sovranatanti colturali sono illustrati nella Tabella 1 . In generale, le positività più elevate sono state riscontrate per il paziente SA, sia prima che dopo coltura. Le percentuali complessive di PCR-positività ottenute sui campioni di SF prima della coltura sono risultate maggiori (62.5\%) ma non statisticamente significative $(P>0.05)$ rispetto a quelle riscontrate sui PBMC (58.3\%). Per i campioni di SF e PBMC analizzati dopo coltura, le percentuali di DNA positività riscontrate dopo $144 \mathrm{~h}$ sono risultate inferiori rispetto a quelle ottenute dopo $72 \mathrm{~h}$. Tali differenze sono apparse statisticamente significative $(P<0.05)$.

L'IF effettuata sui SF e PBMC prima della coltura è risultata negativa su tutti i campioni; dopo la coltura, la valutazione in IF è risultata positiva per C. pneumoniae su 3 campioni di $\mathrm{SF}$ a $72 \mathrm{~h}$ ed a 144h (2 ed 1, rispettivamente) e su 2 campioni di PBMC a 72 h e 144 h (corrispondenti a 2 pazienti differenti).

Nel paziente affetto da SA, l'analisi chimico-fisica del SF ha mostrato un pattern flogistico, con predominanza di cellule polimorfonucleate e assenza di cristalli, mentre le analisi microbiologiche erano negative per germi comuni, C. trachomatis, anaerobi e miceti. Non sono stati identificati inoltre micoplasmi all'esame colturale. Le indagini sierologiche per virus, batteri e parassiti sono risultate negative per infezioni recenti.

I risultati della determinazione delle IgA ed IgG anti-Chlamydophila hanno evidenziato valori elevati (36 e 54 BU, rispettivamente). Sia nel paziente SA che negli altri pazienti esaminati, le colture per tutti i microrganismi testati sono risultate negative. I risultati delle indagini molecolari (PCR ed RT-PCR) effettuate sui SF e PBMC del paziente SA sono esposti nella Figura I (pannelli $a-d$ ). 
Tabella I: Risultati della PCR e dell'isolamento colturale di C. pneumoniae nei pazienti studiati.

\begin{tabular}{|c|c|c|c|c|c|c|c|}
\hline \multirow{3}{*}{$\begin{array}{l}\text { Pazienti } \\
\text { n. }\end{array}$} & \multirow[t]{3}{*}{ DIAGNOSI } & \multicolumn{2}{|c|}{$\begin{array}{c}\text { †PCR } \\
\text { n. positivi/totale }\end{array}$} & \multicolumn{4}{|c|}{$\begin{array}{c}\text { \#PCR } \\
\text { n. positivi/totale }\end{array}$} \\
\hline & & & & $72 \mathrm{~h}$ & I44h & $72 \mathrm{~h}$ & 144h \\
\hline & & SF & PBMC & \multicolumn{2}{|c|}{ SF } & \multicolumn{2}{|c|}{ PBMC } \\
\hline I & Spond. anchilosante & $3 / 3$ & $4 / 7$ & $2 / 3$ & $1 / 3$ & $2 / 7$ & $4 / 7$ \\
\hline $2-3$ & Artrite psoriasica & $1 / 2$ & $1 / 2$ & $1 / 2$ & $0 / 2$ & $0 / 2$ & $0 / 2$ \\
\hline 4 & Gotta & $0 / 1$ & $1 / 1$ & $1 / 1$ & 0 & $0 / 1$ & $0 / 1$ \\
\hline $5-6$ & Gonidrarto di ndd & $1 / 2$ & $1 / 2$ & $1 / 2$ & $0 / 2$ & $0 / 2$ & $0 / 2$ \\
\hline
\end{tabular}

$\dagger$ campioni positivi prima della coltura

\# campioni positivi dopo coltura

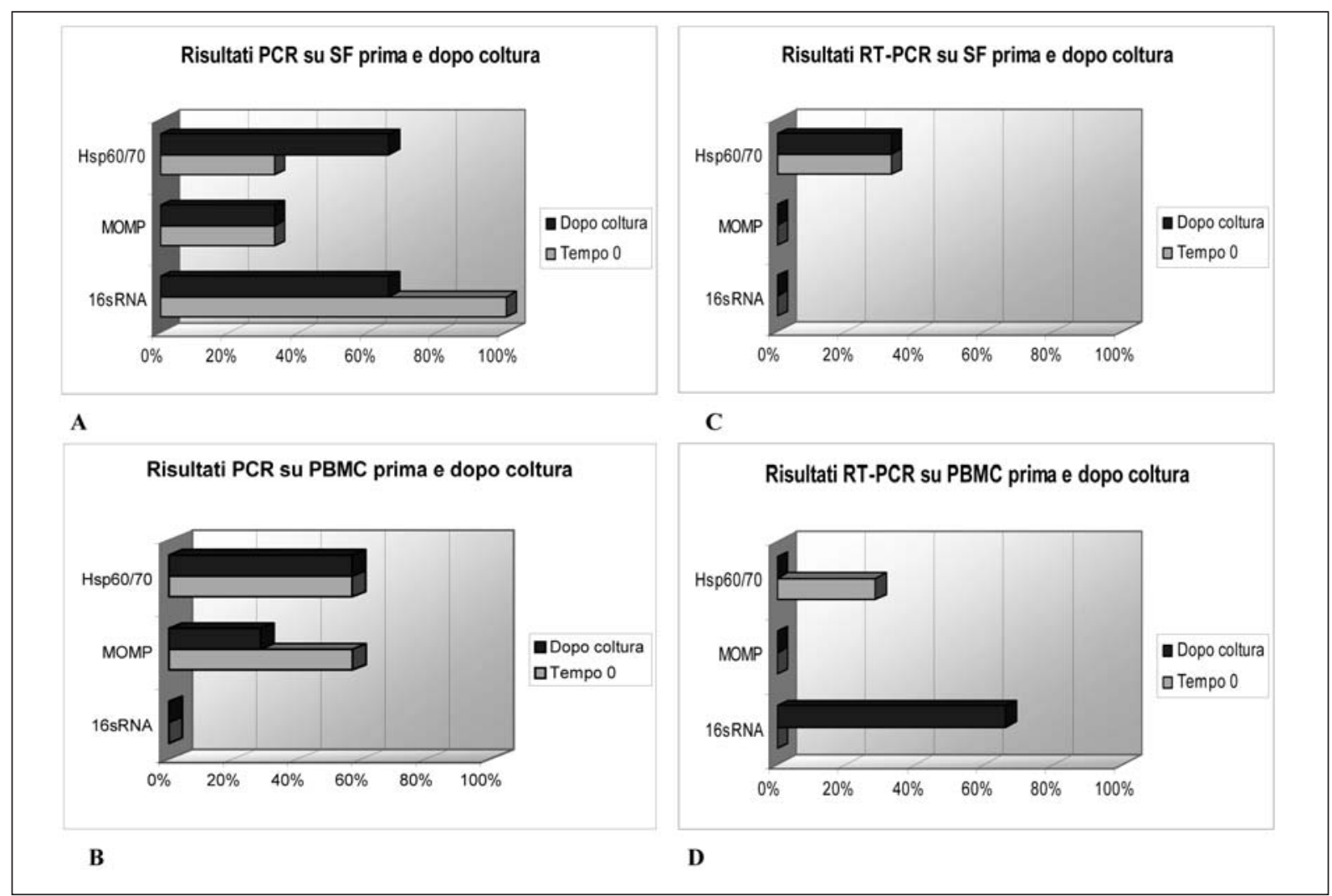

Figura I. Risultati della PCR (pannelli A e B) e della RT-PCR (pannelli $C$ e D) effettuate su SF e PBMC del paziente con SA prima e dopo coltura su Hep2

\section{DISCUSSIONE}

Chlamydophila pneumoniae è un patogeno intracellulare responsabile prevalentemente di infezioni a carico dell'apparato respiratorio. Un suo ruolo tuttavia è stato chiamato in causa anche in alcune patologie acute e croniche dell'apparato cardiovascolare, osteoarticolare e del sistema nervoso. Effettivamente, da qualche anno numerosi laboratori hanno dimostrato la presenza a livello sinoviale di DNA batterico e/o antigeni non tradizionalmente associati alla malattia articolare, relativi a C. pneumoniae. Per tale patogeno, indagini molecolari e una nostra personale casistica (dati non pubblicati) hanno confermato la presenza di C. pneumoniae-DNA in un'ampia percen- tuale di SF e PBMC di pazienti con ReA; tuttavia tali riscontri non hanno tuttora definito una chiara correlazione patogenetica tra presenza di $C$. pneumoniae ed infiammazione sinoviale.

In questo studio abbiamo verificato la presenza di C. pneumoniae-DNA nei SF di pazienti affetti da forme di sinovite cronica che non rispondevano ai criteri diagnostici di ReA: questo risultato appare in linea con la letteratura, ove la presenza di DNA batterico a livello sinoviale (e talvolta DNA di più specie batteriche contemporaneamente) è stata dimostrata in circa il $10 \%$ di pazienti con varie forme di artrite (10).

Il riscontro di DNA nei PBMC dei pazienti studiati ed i risultati ottenuti mediante le indagini 
colturali sembrerebbero suggerire un ruolo patogenetico di C. pneumoniae anche in sinoviti croniche non ReA: C. pneumoniae, eludendo i meccanismi di difesa dell'ospite, sarebbe in grado di sopravvivere all'interno delle cellule mononucleate e verrebbe trasportata a distanza verso siti periferici, tra cui il tessuto sinoviale, ove può essere riscontrata in una forma vitale in grado di propagarsi in altri tipi cellulari (Hep2) (5).

Per il paziente con SA, giunto alla nostra osservazione a causa di una variazione delle caratteristiche cliniche della patologia di base, l'obiettività e le indagini chimico-fisiche e colturali effettuate sul SF indirizzavano la diagnosi verso una forma di artrite infettiva acquisita di recente. Per questo paziente $C$. pneumoniae è risultato l'unico patogeno sinoviale. I sequenziamento dei prodotti di amplificazione effettuato per l'analisi statistica delle omologie (BLAST) hanno confermato la specificità delle metodica (dati non esposti).

Le indagini sierologiche, escludendo il coinvolgimento di microorganismi tradizionalmente coinvolti nella patogenesi delle ReA, hanno confer- mato tale positività. Il ruolo patogenetico di $C$. pneumoniae inteso soprattutto nell'ambito della clinical persistence, sembrerebbe in questo caso testimoniato oltre che dall'assenza di $\operatorname{IgM}$, dall'elevata concentrazione sierica di IgA ed IgG specifiche, indicativa di possibile reinfezione da $C$. pneumoniae (7).

I dati ottenuti con l'RT-PCR indicano che i geni codificanti per le proteine Hsps di C. pneumoniae, fortemente implicate nella genesi del danno tissutale, sono trascritti sia nei PBMC periferici che in sinovia, e in quest'ultima sia prima che dopo coltura; il mancato riscontro di $H s p$-mRNA nei PBMC dopo la coltura farebbe presupporre la cessazione dell'attività metabolica del microrganismo. Viceversa, la selettiva attenuazione di MOMP e 16sRNA in entrambi i compartimenti, in linea con alcuni recenti dati della letteratura (9), lascia ipotizzare una differente espressione di geni durante le varie fasi dell'infezione. Ulteriori studi sono in corso per verificare la correlazione patogenetica tra $C$. pneumoniae (in stato di persistenza o di attiva crescita) e flogosi sinoviale.

Tabella I: Risultati dell'isolamento colturale di C. pneumoniae nei pazienti studiati

\begin{tabular}{|c|c|c|c|c|c|c|c|}
\hline \multirow{4}{*}{$\begin{array}{c}\begin{array}{c}\text { Pazienti } \\
\text { n. }\end{array} \\
\text { I }\end{array}$} & \multirow{4}{*}{$\begin{array}{c}\text { DIAGNOSI } \\
\text { Spond. anchilosante }\end{array}$} & \multirow{2}{*}{\multicolumn{2}{|c|}{$\begin{array}{c}\text { n. di campioni PCR } \\
\text { positivi/totale prima della } \\
\text { coltura }\end{array}$}} & \multicolumn{4}{|c|}{ n.di campioni PCR positivi/totale dopo coltura } \\
\hline & & & & $72 \mathrm{~h}$ & I44h & $72 \mathrm{~h}$ & I44h \\
\hline & & \multirow{2}{*}{$\begin{array}{l}\text { SF } \\
3 / 3\end{array}$} & \multirow{2}{*}{$\begin{array}{c}\text { PBMC } \\
4 / 7\end{array}$} & \multicolumn{2}{|c|}{ SF } & \multicolumn{2}{|c|}{ PBMC } \\
\hline & & & & $2 / 3$ & $1 / 3$ & $2 / 7$ & $4 / 7$ \\
\hline 2 & Artrite psoriasica & $1 / \mathrm{I}$ & $1 / 1$ & $\mathrm{I} / \mathrm{I}$ & $1 / 1$ & $\mathrm{I} / \mathrm{I}$ & 0 \\
\hline 3 & Artrite psoriasica & $1 / 1$ & $1 / 1$ & $\mathrm{I} / \mathrm{I}$ & $\mathrm{I} / \mathrm{I}$ & $\mathrm{I} / \mathrm{I}$ & 0 \\
\hline 5 & Gotta & 0 & $\mathrm{I} / \mathrm{I}$ & $1 / \mathrm{I}$ & 0 & $\mathrm{I} / \mathrm{I}$ & 0 \\
\hline 5 & Gonidrarto di ndd & $1 / 1$ & $1 / 1$ & $1 / 1$ & $1 / 1$ & 0 & $1 / \mathrm{I}$ \\
\hline 6 & Gonidrarto di ndd & 0 & 0 & 0 & 0 & 0 & 0 \\
\hline \multicolumn{2}{|c|}{ Totale positività (\%) } & 75 & 67 & 75 & 50 & 42 & 42 \\
\hline
\end{tabular}

Tabella 2: Risultati della PCR e RT-PCR su SF e PBMC di un paziente con SA prima e dopo coltura su Hep2.

\begin{tabular}{|c|c|c|c|c|c|c|c|}
\hline \multicolumn{2}{|c|}{ Campioni } & \multicolumn{2}{|c|}{ n. (\%) di campioni PCR positivi } & \multicolumn{3}{c|}{ n. (\%) di campioni RT- PCR positivi } \\
\cline { 3 - 8 } & I6sRNA & MOMP & HsP-60 & I6sRNA & MOMP & HsP-60 \\
\hline \multirow{2}{*}{ SF (n.3) } & p.c. & 66 & 33 & 66 & 0 & 0 & 33 \\
\hline $\begin{array}{c}\text { PBMC } \\
\text { (n.7) }\end{array}$ & d.c. & 100 & 33 & 33 & 0 & 0 & 33 \\
\hline
\end{tabular}

p.c. $=$ prima della coltura; d.c. $=$ dopo coltura 


\section{BILIOGRAFIA}

1. Altun S, Kasapcopur O, Aslan M, et al. Is there any relationship between Clamydophila pneumoniae infection and juvenile idiopathic arthritis? J Med Microbiol 2004; 53: 787-90.

2. Braun J, Kingsley G, van der Heijde D, et al. On the difficulties of establishing a consensus on the definition of and diagnostic investigation for reactive arthritis. Results and discussion of a questionnaire prepared for the 4th International Workshop on Reactive Arthritis, Berlin, Germany, July 3-6, 1999. J Rheumatol 2000; 27: 2185-92.

3. Carty SM, Snowden N, Silman AJ. Should infection still be considered as the most likely triggering factor for rheumatoid arthritis? J Rheumatol 2003; 30: 4259.

4. Contini C, Cultrera R, Seraceni S, Castellazzi M, Granieri E, Fainardi E. Cerebrospinal fluid molecular demonstration of Chlamydia pneumoniae DNA is associated to clinical and brain MRI activity in a subset of patients with relapsing remitting multiple sclerosis. Mult Scler. 2004; 10: 360-9.

5. Contini C, Seraceni S, Giuliodori M, et al. Successful isolation of $C$. pneumoniae from synovial fluids and blood cultures of a patient with undifferentiated spondyloarthropathy. Int J Inf Dis: Abstract $n^{\circ} 3010$.

6. Cunningham AF, Johnson SL, Julious SA, et al. Chronic Chlamydia pneumoniae infection and asthma exacerbations in children. Eur Resp J 1998; 11: 3459.

7. Falck G, Engstrand I, Gad A, Gnarpe J, Gnarpe H, Laurila A. Demonstration of Chlamydia pneumoniae in patients with chronic pharyngitis. Scand J Infect Dis 1997; 29: 585-9.

8. Gaydos CA, Quinn TC, Eiden JJ. Identification of Chlamydia pneumoniae by DNA amplification of the 16sRNA gene. J Clin Microbiol 1992; 30: 796-800.

9. Gerard H, Schumacher HR, El-Gabalawy H, Goldbach-Mansky R, Hudson AP. C. pneumoniae present in the human synovium are viable and metabolically active. Microb Pathogen 2000; 29: 17-24.

10. Gerard HC, Wang Z, Wang GF. Chromosomal DNA from a variety of bacterial species is present in synovial tissue from patients with various forms of arthritis. Arthritis Rheum 2001; 44(7): 1689-97.

11. Hannu T, Puolakkainen M, Leirisalo-Repo M. Chlamydia pneumoniae as triggering agent in Reactive arthritis. Rheumatology 1999; 38: 411-4.

12. Kwok S, Higushi R. avoiding false positives with PCR. Nature 1989; 339: 237-8.

13. Mahony JB, Chonh BK, Coombes M, et al. Analytical sensitivity, reproducibility of results and clinical performance of five PCR assays for detecting Chlamydia pneumoniae DNA in peripheral blood mononuclear cells. J Clin Microbiol 2000; 38: 2622-7.

14. Rhil M, Kohler L, Klos A, et al. Persistent infection of Chlamydia in reactive arthritis. Ann Rheum Dis 2006; 65: 281-4.

15. Sambrook J, Fritsch EF, Maniatis T. molecular cloning: a laboratory manual, $2^{\text {nd }}$ ed. Cold Spring Harbor Laboratory Press, NY. 1989; pp E3-E14.

16. Shumacker HR jr, Gerard HC, Arayssi T, et al. Lower prevalence of Chlamydia pneumoniae DNA compared with Chlamydia trachomatis DNA in synovial tissue of arthritis patients. Arthritis Rheum 1999; 42: 1889-93.

17. Sieper J, Braun J. Pathogenesis of spondyloarthropathies. Arthritis Rheum 1995; 38: 1547 - 54.
18. Villareal C, Whittum-Hudson J, Hudson A, et al. Persistent Chlamydiae and chronic arthritis. Arthritis Res 2002; 4: 5-9.

\section{Ringraziamenti}

Lavoro realizzato in parte grazie a contributi PRIN 2005-2006, Fondazione CARIFE e CARICE 2005-2006. Si ringrazia la Prof. A. DautryVarsat dell'Istituto Pasteur di Parigi per la sua preziosa assistenza.

Un ringraziamento va inoltre al Dr. Matteo Colina della Sezione di Reumatologia dell'Università di Ferrara per averci in parte fornito i campioni biologici dei pazienti studiati.

\section{Carlo Contini}

Università di Ferrara, Sezione di Malattie Infettive, Dipartimento di Medicina Clinica e Sperimentale Tel.: 0532 291310; E-mail: cnc@unife.it 